\title{
Estructura de tallas de captura y relación longitud-peso de peces nativos en el humedal Abras de Mantequilla, Ecuador
}

\section{Structure of catch sizes and length-weight ratio of native fish in the Abras de Mantequilla wetland, Ecuador}

Berta Yadira Ochoa Ubilla ${ }^{1}$, Kléver Xavier Mendoza Nieto ${ }^{2}$, Roque Vivas Moreira ${ }^{3}$, ${ }^{\triangle}$ Juan Urdánigo Zambrano ${ }^{4}$, Yarelys Ferrer-Sánchez ${ }^{4}$

${ }^{1}$ Viceministerio de Acuacultura y Pesca del Ecuador. Inspectoría de Pesca Provincia de Los Ríos. Calle Sucre y General Barona, Malecón.Babahoyo Ecuador: yadira.ochoa@pesca.gob.ec

${ }^{2}$ Universidad Laica "Eloy Alfaro" de Manabi. Facultad Ciencias del Mar. Laboratorio de Biología Pesquera. Ciudadela Universitaria vía a San Mateo, Manta Ecuador: klever.mendoza@uleam.edu.ec

${ }^{3}$ Universidad Técnica Estatal de Quevedo. Unidad de Posgrado, Campus "Ingeniero Manuel Haz Álvarez”, Av. Quito, km 1.5 vía a Santo Domingo de los Tsáchilas.EC.120301.Quevedo,Ecuador: rvivas@uteq.edu.ec

${ }^{4}$ Universidad Técnica Estatal de Quevedo. Facultad de Ciencias Ambientales. Carrera de Ingeniería en Gestión Ambiental. Campus “Ingeniero Manuel Haz Álvarez”, Av. Quito, km 1.5 vía a Santo Domingo de los Tsáchilas. EC.120301.

Quevedo,Ecuador: jurdanigo@uteq.edu.ec; yferrer@uteq.edu.ec

Rec.: 3.03.2016. Acept.: 30.06.2016.

Publicado el 30 de diciembre de 2016

\section{Resumen}

$\mathrm{E}_{\mathrm{c}}^{\mathrm{se}}$ ste estudio tuvo como objetivos identificar la composición de capturas, describir la estructura de tallas y determinar la relación longitud-peso de las principales especies de peces nativos de interés comercial del humedal Abras de Mantequilla (AdM). Durante los meses de abril a septiembre de 2015 se recolectaron aleatoriamente 492 peces provenientes de los desembarques del humedal AdM y se midió la longitud total $(\mathrm{Lt})$ y el peso total $(\mathrm{Pt})$ a cada ejemplar. Para la identificación taxonómica de las especies se utilizaron las claves, guías y catálogos especializados. La estructura de tallas se analizó mediante histogramas de frecuencia, se compararon las tallas medias de capturas (TMC) con la talla de comercialización permitida (TCP) y la talla media de madurez (TMM). La relación longitud-peso se estimó mediante la ecuación de alometría de Ricker. La especie más abundante fue Ichthyolephas humeralis seguida por Pseudocurimata spp., mientras que Leporinus ecuadoriensis fue la especie menos capturada. Rhamdia cinerascens presentó una TMC de $24.81 \mathrm{~cm}(\mathrm{Lt})$, inferior a la talla media de madurez $(25 \mathrm{~cm} \mathrm{Lt})$. Ichthyolephas humeralis, Andinoacara rivulatus y Pseudocurimata spp., presentaron crecimiento alométrico negativo, y el resto de especies tuvieron crecimiento isométrico. En la mayoría de las especies de peces el reclutamiento ocurrió a una edad y tamaño óptimo en la que los peces ya aportaron con nuevos individuos a la población. Se evidenció que existe una relación entre las especies con crecimiento alométrico negativo y su abundancia en las capturas.

Palabras clave: reclutamiento, alométrico, isométrico, léntico, lótico, ictiofauna.
The objective of this study was to identify the composition of catches, to describe the size structure and to determine the length-weight ratio of the main species of native fish of commercial interest in the Abras de Mantequilla wetland (AdM). During April to September, 492 fishes from the wetland AdM were randomly collected, the total length (Tl) and the total weight (Tw) were measured for each specimen. For the taxonomic identification of the species the keys, guides and specialized catalogs were used. The size structure was analyzed by frequency histograms, the mean catch sizes (MCZ) were compared with permitted commercial size (PCS) and mean maturity size (MMS). The length-weight relationship was estimated using the Ricker allometry equation. The most abundant species was Ichthyolephas humeralis followed by Pseudocurimata spp., while Leporinus ecuadoriensis was the least captured species. Rhamdia cinerascens presented a MCZ of $24.81 \mathrm{~cm}(\mathrm{Tl})$, inferior to the average size of maturity $(25 \mathrm{~cm} \mathrm{Tl})$. Ichthyolephas humeralis, Andinoacara rivulatus and Pseudocurimata spp. presented negative allometric growth, while the rest of species have isometric growth. In most fish species recruitment occurred at an optimal age and size in which the fish already contributed with new individuals to the population. It was evidenced that there is a relation between the species with negative allometric growth and its abundance in the catches.

Key words: recruitment, allometric, isometric, lentic, lotic, ichthyofauna. 


\section{Introducción}

Los humedales asociados a los hábitats de ríos tropicales poseen una diversa flora y fauna con alto grado de especialización (Boavida, 1999; Quevedo, 2008). Son importantes corredores longitudinales y transversales para la dispersión de la biota. Las funciones de los humedales son diversas, incluyendo la retención de agua, control de inundaciones, purificación de agua, provisión de recursos pesqueros y forestales (AlvarezMieles et al., 2013).

El humedal Abras de Mantequilla (AdM) ubicado en la provincia Los Ríos, es blanco de una serie de presiones de origen humano, las cuales incluyen la extracción de agua para riego y consumo, deforestación, intensificación agrícola y explotación pesquera (Quevedo, 2008; Alvarez-Mieles et al., 2013). En este humedal, la pesca es artesanal y de subsistencia, los peces capturados son almacenados vivos en copos para su posterior consumo por parte de los pescadores y el excedente es destinado a la comercialización local y regional (Freitas \& Rivas, 2006; Revelo \& Castro, 2010). Los pescadores realizan sus faenas de pesca en horas de la tarde, empleando artes de pesca tales como red de enmalle (monofilamento y multifilamento) y red o paño de multifilamento utilizado en la modalidad "tape de espera o estático" con túnel y copo. Las capturas generalmente están compuestas por especies nativas como Bocachico (Ichthyoelephas humeralis), Vieja azul (Andinoacara rivulatus), Guanchiche (Hoplias microlepis), Barbudo (Rhamdia cinerascens), Ratón (Leporinus ecuadoriensis), Dama (Brycon sp.) y Dica (Pseudocurimata spp.) (Revelo \& Castro, 2010).

Las primeras investigaciones sobre peces de aguas continentales en la provincia Los Ríos fueron los trabajos de: Barhill \& López-León (1973) que estudiaron la biología de peces en el río Vínces; Cadena (1981) que realizó un estudio de la relación longitud-peso de I. humeralis; Florencio \& Álvarez (1983), quienes realizaron estudios bioecológicos y de madurez sexual de I. humeralis. El Instituto Nacional de Pesca (INP), durante los últimos años, ha realizado varios trabajos técnicos en Los Ríos relacionados con la actividad pesquera, calidad de agua y aspectos reproductivos (Villón et al., 1999; Revelo \& Elías, 2004; Prado et al., 2010).

Posteriormente, el INP publicó una evaluación de aspectos biológicos y pesqueros en la provincia Los Ríos (Revelo, 2010; Revelo \& Castro, 2010), un catálogo de peces de aguas continentales en la provincia (Revelo \& Laaz, 2012) y un estudio formal acerca de los parámetros biológicos de Pseudocurimata boulengeri en el embalse Chongón (Chicaiza \& Flores, 2016). En vista de la información generada, la Subsecretaria de Recursos Pesqueros del Ecuador (SRP), creó el Acuerdo Ministerial 022-A (AM 022-A), con la finalidad de ordenar la actividad pesquera en la provincia Los Ríos, estableciendo artes de pesca (redes) con ojo de malla estirado de 2" y tallas de captura. Sin embargo, las tallas de captura establecidas presentan inconvenientes al momento de su aplicación, debido a que el arte de pesca más empleado (red) tiene una selectividad homogénea y los peces poseen tipos de crecimiento diferentes entre sí.

Aunque existen varios estudios sobre peces nativos en la provincia Los Ríos, hasta ahora es escaso el conocimiento que se tiene en relación a la composición de las capturas, tallas de captura y relación longitud-peso, sobre todo en los peces del humedal AdM. Teniendo en cuenta esta situación, este estudio tuvo como objetivos identificar la composición de capturas, describir la estructura de tallas y determinar la relación longitud-peso de las principales especies de peces nativos de interés comercial del humedal Abras de Mantequilla. Con este estudio se esperarían observar tallas de captura superiores a la talla de madurez y también un crecimiento del tipo isométrico de los peces nativos. Esta información servirá de base para el diseño de estrategias de conservación y de ordenamiento pesquero en el humedal AdM.

\section{Materiales y métodos}

\section{Área de estudio}

El humedal Abras de Mantequilla (AdM) $(56,000$ ha) está ubicado en la zona central de la provincia Los Ríos (1²8' 0" S; 79 34' 59” W). Fue reconocido como sitio RAMSAR en el 2000, debido a su relevancia como base de anidamiento para especies migratorias ornitológicas y por su fauna ictiológica nativa. El humedal AdM está constituido por depresiones naturales de terrenos alimentadas por aguas procedentes de la precipitación pluvial y del aporte hídrico de ríos y esteros con elevaciones entre 5-10 m (Quevedo, 2008; BirdLife International, 2016).

Se recolectaron 492 muestras mediante muestreo aleatorio a partir de la pesca proveniente del humedal, durante los meses de abril a septiembre de 2015 (temporada de actividad pesquera). A cada individuo se le midió la longitud total (Lt) con un ictiómetro de $0.1 \mathrm{~cm}$ de precisión y el peso total $(\mathrm{Pt})$ con una balanza digital de $0.10 \mathrm{~g}$ de precisión. La información se organizó en hojas de registro y hojas de cálculo en Excel, procesadas mediante el software estadístico InfoStat.

\section{Composición de la captura}

Para la identificación taxonómica de las especies 
se utilizaron las claves, guías y catálogos de Eigenmann \& Allen (1942), Fischer et al. (1995), Hilderbrand \& Kershner (2004), Nava-Flores \& Ancona (2010) y Revelo \& Laaz (2012). La composición de capturas se estimó por la variación de la abundancia relativa del total de las especies capturadas en los diferentes sitios de pesca. Para esto se consideró como $100 \%$ al total de organismos capturados durante el periodo de muestreo.

\section{Estructura de tallas}

La estructura de tallas se analizó mediante histogramas de frecuencia. Se utilizó la prueba Kolmogorov-Smirnov (Zar, 2010), para comparar las distribuciones de tallas de cada especie. Posteriormente, se compararon las tallas medias de capturas (TMC) con longitudes inferiores a la talla de comercialización permitida (TCP) del AM 022-A y con la talla media de madurez sexual (TMM) publicadas en el informe de Revelo (2010).

\section{Relación longitud-peso}

La relación longitud-peso se estimó mediante la ecuación de alometría de Ricker (1975):

$$
\mathrm{W}=\mathrm{aLt} \mathrm{t}^{\mathrm{b}}
$$

Donde:

$\mathrm{W}$ es el peso total en $\mathrm{g}$

Lt la longitud total del pez en $\mathrm{cm}$

a es la ordenada de origen y la pendiente b se utiliza para describir el tipo de crecimiento que exhibe el pez.

Se considera crecimiento isométrico $\mathrm{si} b=3$, cuando el peso y longitud crecen en la misma proporción con el tiempo, si b>3 el crecimiento se considera alométrico positivo, y será alométrico negativo si $b<3$ (Cifuentes et al., 2012; Chicaiza \& Flores, 2016). El grado de asociación entre las variables longitud-peso fue calculado por el coeficiente de determinación $\mathrm{R}^{2}$.

Para evaluar si los coeficientes de alometría (b) obtenidos fueron significativamente diferentes de 3 , se aplicó la prueba t de Student (Zar, 2010), utilizando el siguiente estadístico:

$$
\mathrm{t}=(\mathrm{b}-\beta) / \mathrm{S}
$$

Donde:

$\mathrm{t}$ es $\mathrm{t}$ de Student,

b es el coeficiente de alometría,

$\beta$ es la pendiente de referencia $(=3)$

S el error estándar.

La hipótesis a probar fue: Ho: $b=3$ (crecimiento isométrico). Ha: $b \neq 3$ (crecimiento alométrico).

\section{Resultados y discusión}

\section{Composición de capturas}

En las 492 muestras analizadas (Cuadro 1), Ichthyoelephas humeralis fue la especie más abundante (37\%) y Leporinus ecuadoriensis la menos representativa (6\% de las capturas; Figura 1). Estos resultados difieren de lo descrito por Pacheco (2013, 2014, 2015) y Revelo (2010) donde Pseudocurimata spp., representó la especie de mayor captura $(37.70,30.20,25.20$ y $42.30 \%$, respectivamente). Las diferencias en la composición de las capturas podrían estar relacionadas con la amplia distribución y la importancia comercial de Pseudocurimata spp. en el ambiente léntico del embalse Chongón (Chicaiza \& Flores, 2016).

Cuadro 1. Peces de interés comercial capturados en el humedal Abras de Mantequilla, Ecuador

\begin{tabular}{lll}
\hline \multicolumn{1}{c}{ Familia } & \multicolumn{1}{c}{ Nombre científico } & \multicolumn{1}{c}{$\begin{array}{c}\text { Nombre } \\
\text { comercial }\end{array}$} \\
\hline Prochilodontidae & Ichthyolephas humeralis & Bocachico \\
Cichlidae & Andinoacara rivulatus & Vieja Azul \\
Erythrinidae & Hoplias microlepis & Guanchiche \\
Heptapteridae & Rhamdia cinerascens & Barbudo \\
Bryconidae & Brycon sp. & Dama \\
Anostomidae & Leporinus ecuadoriensis & Ratón \\
Curimatidae & Pseudocurimata spp. & Dica \\
\hline
\end{tabular}

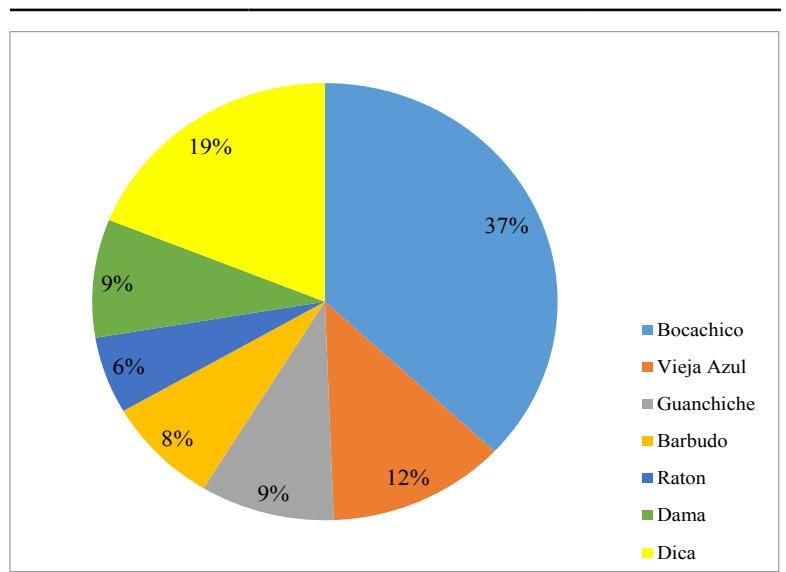

Figura 1. Porcentaje de capturas de las principales especies de peces nativos en el humedal Abras de Mantequilla, Ecuador

\section{Estructura de tallas}

La distribución de frecuencia de tallas mostró que todas las especies presentaron TMC mayores a las TCP reguladas en el Acuerdo Ministerial 022-A (Cuadro 2). Brycon sp. presentó una TMC de $24.91 \mathrm{~cm}$ (Lt) (Figura 2e), superior a la talla media de captura $15.90 \mathrm{~cm}(\mathrm{Lt})$ 
Cuadro 2. Parámetros estadísticos de la estructura de tallas de las principales especies de peces de interés comercial, capturadas en el humedal Abras de Mantequilla, Ecuador, durante 2015. Talla media de captura (TMC); talla de captura permitida (TCP); cantidad de individuos (N); desviación estándar (DS)

\begin{tabular}{lcccccc}
\hline \multicolumn{1}{c}{ Especie } & N & Rango & DS & TMC & TCP & p \\
\hline Ichthyolephas humeralis & 182 & $17-35$ & 3.24 & 22.83 & 20 & $<0.001$ \\
Andinoacara rivulatus & 61 & $15-22$ & 1.8 & 18.03 & 13 & $<0.001$ \\
Hoplias microlepis & 46 & $19-35$ & 3.93 & 27.38 & 20 & $<0.001$ \\
Rhamdia cinerascens & 39 & $21-29$ & 1.79 & 24.81 & 20 & $<0.001$ \\
Brycon sp. & 43 & $18-30$ & 2.86 & 23.83 & 20 & $<0.001$ \\
Leporinus ecuadoriensis & 28 & $19-29$ & 3.19 & 24.91 & 19 & $<0.001$ \\
Pseudocurimata spp. & 93 & $14-26$ & 2.56 & 19.53 & 16 & $<0.001$ \\
\hline
\end{tabular}

reportada por Rivera (2014) en el río Mira al norte de Ecuador, y menor a la TMC y TMM reportada por Pacheco \& Cabanilla (2013) en el embalse Chongón con 29.20 y $29.50 \mathrm{~cm}(\mathrm{Lt})$ respectivamente.

Ichthyolephas humeralis presentó frecuencias de tallas en los rangos comprendidos desde $17-35 \mathrm{~cm}$ (Lt) (Figura 2a), inferiores a lo reportado por Revelo \& Castro (2010), quienes encontraron individuos con tallas entre 16-37 cm (Lt). A pesar de lo descrito, la TMC se situó por encima de la TMM, lo cual es favorable ya que el $88 \%$ de los individuos ya ha realizado su desove.

Leporinus ecuadoriensis obtuvo frecuencias de tallas comprendidas entre 19 y $29 \mathrm{~cm}$ de longitud total, la TMC fue $23.83 \mathrm{~cm}(\mathrm{Lt})$ y el $100 \%$ de las capturas fueron superiores a la TMM (Figura 2f). Resultados distintos para Leporinus muyscorum fueron descritos por Bru, Segura, \& Olaya (2009) quienes determinaron una TMC de $25.8 \mathrm{~cm}(\mathrm{Lt})$ superior a lo registrado en este estudio. No obstante, a pesar de lo descrito para L. muyscorum en el río Síu Colombia, esta pesquería presenta problemas de sobrepesca al reclutamiento y al crecimiento debido a que es sustentada por individuos muy pequeños y muy jóvenes.

Las especies $A$. rivulatus, $H$. microlepis y Pseudocurimata spp. presentaron TMC de 18.03, 27.38 y $19.53 \mathrm{~cm}(\mathrm{Lt})$ respectivamente (Figura 2b, 2c y $2 \mathrm{~g}$ ), menores a las tallas registradas por Chicaiza \& Flores (2016) y Pacheco \& Cabanilla (2013) con 19.10, 30.40 , y $24.40 \mathrm{~cm}(\mathrm{Lt})$ respectivamente en el embalse Chongón. Esto puede deberse a que en los sistemas fluviales lénticos del humedal AdM existe una mayor sobrexplotación de las especies ícticas más apreciadas como bocachico, vieja azul y dica, deterioro ambiental provocado por la agricultura, industria, minería y tala de bosques, convirtiéndose en posibles causas del deterioro de las pesquerías de los sistemas fluviales de América (FAO, 2004).

Asimismo, resultados distintos presentaron Gámez et al. (2014) quienes registraron para Aequidens pulcher una talla media de $10.40 \mathrm{~cm}(\mathrm{Lt})$ inferior a la TMC $(18.03$ $\mathrm{cm} \mathrm{Lt)} \mathrm{registrada} \mathrm{en} \mathrm{este} \mathrm{estudio} \mathrm{para} A$. rivulatus. Las diferencias en las TMC podrían ser consecuencia del interés socioeconómico de los pobladores de la Ciénaga Grande de Santa Marta, Colombia, debido a que estos viven directamente de la pesca, ejerciendo un gran esfuerzo pesquero sobre $A$. pulcher.

Balboni et al. (2011) registraron una talla media de $48.79 \mathrm{~cm}(\mathrm{Lt})$ para Hoplias aff. malabaricus en el lago Yalca Argentina, superior a lo registrado en este estudio. Las diferencias entre las tallas medias podrían ser resultado de la ausencia de presión pesquera en el lago Yalca desde hace ocho años. Además, los resultados del estudio difieren de lo registrado en el Parque Nacional Lagoa do Peixe, Brasil, donde se registra una TMC de $17.73 \mathrm{~cm}(\mathrm{Lt})$ para Hoplias aff malabaricus, probablemente por la importancia de la especie en la pesquería local y el esfuerzo pesquero al que está sometida (Corrêa et al., 2016).

Las TMC de las especies registradas fueron superiores a las TMM descritas en el informe del INP (Revelo, 2010), a excepción de Rhamdia cinerascens que presentó una TMC inferior a la TMM. Es decir, que en la mayoría de las especies el reclutamiento ocurrió a una edad y tamaño óptima en la que los especímenes ya aportaron con nuevos individuos a la población (FAO, 1974).

\section{Relación longitud-peso}

Los valores del coeficiente de determinación $\mathrm{R}^{2}$ mostraron una relación significativa entre las variables de estudio $p>0.05$ para todas las especies (Cuadro 3). El coeficiente de crecimiento b mostró que las especies: Ichthyolephas humeralis y Pseudocurimata spp., presentaron un crecimiento alométrico negativo $(\mathrm{p}=0.017 ; \mathrm{p}=0.007$, respectivamente) (Cuadro 3; figuras $3 \mathrm{a}$ y $3 \mathrm{~g}$ ). Además, Andinoacara rivulatus 

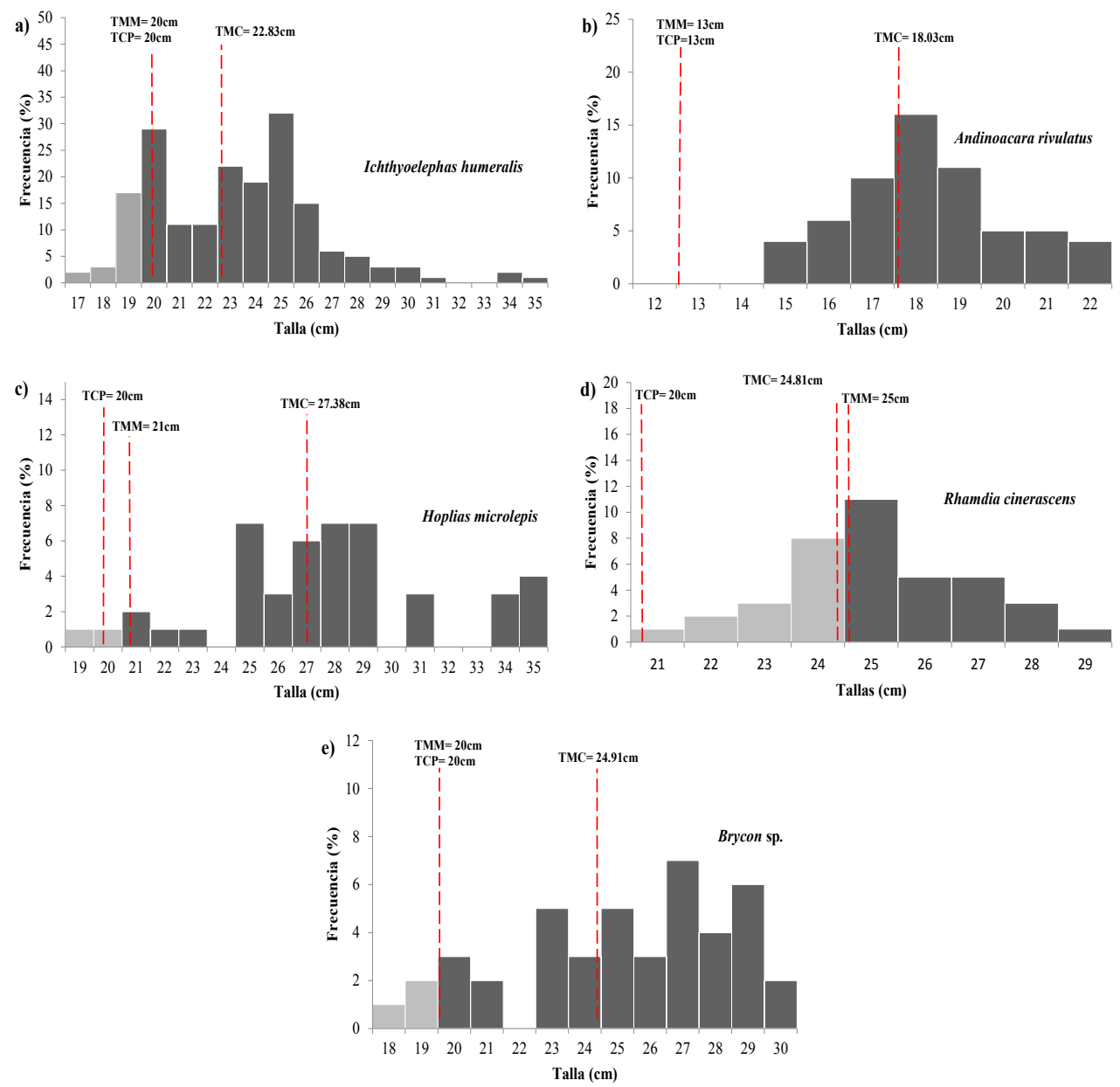

Figura 2. Estructura de tallas de las principales especies capturadas en el humedal Abras de Mantequilla, Ecuador; a) Ichthyolephas humeralis, b) Andinoacara rivulatus, c) Hoplias microlepis, d) Rhamdia cinerascens, e) Brycon sp., f) Leporinus ecuadoriensis, g) Pseudocurimata spp

también obtuvo un valor de $\mathrm{b}=2.74$ y mediante la prueba $t$ se determinó que esta especie tuvo un crecimiento alométrico negativo $\mathrm{p}=0.043$.

La especie $H$. microlepis, presentó un crecimiento isométrico $(\mathrm{b}=3.011 ; \mathrm{p}=0.05)$, de la misma manera que Hauser \& Benedito (2012) reportaron un crecimiento isométrico para Hoplias aff. malabaricus en el río Paraná, Brasil. Para H. microlepis en sistemas lénticos y lóticos de Ecuador, Granda \& Montero (2015) encontraron características alométricas. Esto concuerda con Corrêa et al. (2016) quienes indican que $H$. aff. malabaricus presenta un crecimiento alométrico positivo.

Las especies $R$. cinerascens, Brycon sp. y L. ecuadoriensis presentaron crecimiento de tipo isométrico $(\mathrm{p}=0.32 ; \mathrm{p}=0.31 ; \mathrm{p}=0.08)$. Para Pseudocurimata spp., el modelo potencial de alometría fue $\mathrm{Pt}=0.087 \mathrm{Lt}^{2.373}$ con un coeficiente de determinación entre Lt y Pt de $88 \%$ (Figura $3 \mathrm{~g}$ ). El valor del exponente $b=2.373$ fue significativamente diferente al valor de 3 $(\mathrm{p}<0.05)$, lo que determinó un crecimiento alométrico negativo de la especie. Este resultado fue diferente al reportado por Chicaiza \& Flores (2016) para la especie Pseudocurimata boulengeri $(\mathrm{b}=2.57)$ y para machos y hembras (2.70) con un crecimiento isométrico $(\mathrm{p}>0.05)$ para sexos combinados en el embalse de Chongón de la provincia Guayas, Ecuador.

Las diferencias entre los tipos de crecimiento pueden estar relacionados a muchos factores, como la diferencia en tamaños de las muestras, rangos de tallas de especímenes, diferencias genéticas ente grupos de especies y condiciones ambientales locales (Froese, 2006). De igual manera, Santos, Pessanha, Costa, \& Araújo (2004) indican que los parámetros de la relación talla-peso pueden diferir no solo entre especies sino también entre poblaciones de la misma especie, considerando que el coeficiente de crecimiento depende de las diferencias genéticas, nutricionales y ambientales. 
Cuadro 3. Parámetros de la relación longitud-peso de las principales especies de peces de interés comercial capturadas en el humedal Abras de Mantequilla, Ecuador, durante 2015. Ordenada de origen: a; tipo de crecimiento: b; coeficiente de determinación: $R 2$; $t$ de Student: $t$, alométrico negativo: A-, isométrico: I

\begin{tabular}{lcccccc}
\hline Especie & $\mathbf{a}$ & $\mathbf{b}$ & $\mathbf{R}^{2}$ & $\mathbf{t}$ & $\mathbf{p}$ & Crecimiento \\
\hline Ichthyolephas humeralis & 0.071 & 2.427 & 0.83 & -3.50 & $<0.05$ & $\mathrm{~A}-$ \\
Andinoacara rivulatus & 0.044 & 2.743 & 0.88 & -2.69 & $<0.05$ & $\mathrm{~A}-$ \\
Hoplias microlepis & 0.009 & 3.011 & 0.95 & -0.70 & $>0.05$ & $\mathrm{I}$ \\
Rhamdia cinerascens & 0.009 & 2.969 & 0.88 & -1.19 & $>0.05$ & $\mathrm{I}$ \\
Brycon sp. & 0.010 & 2.992 & 0.92 & -1.12 & $>0.05$ & $\mathrm{I}$ \\
Leporinus ecuadoriensis & 0.025 & 2.702 & 0.94 & -2.35 & $>0.05$ & $\mathrm{I}$ \\
Pseudocurimata spp. & 0.087 & 2.373 & 0.88 & -4.37 & $<0.05$ & $\mathrm{~A}-$ \\
\hline
\end{tabular}
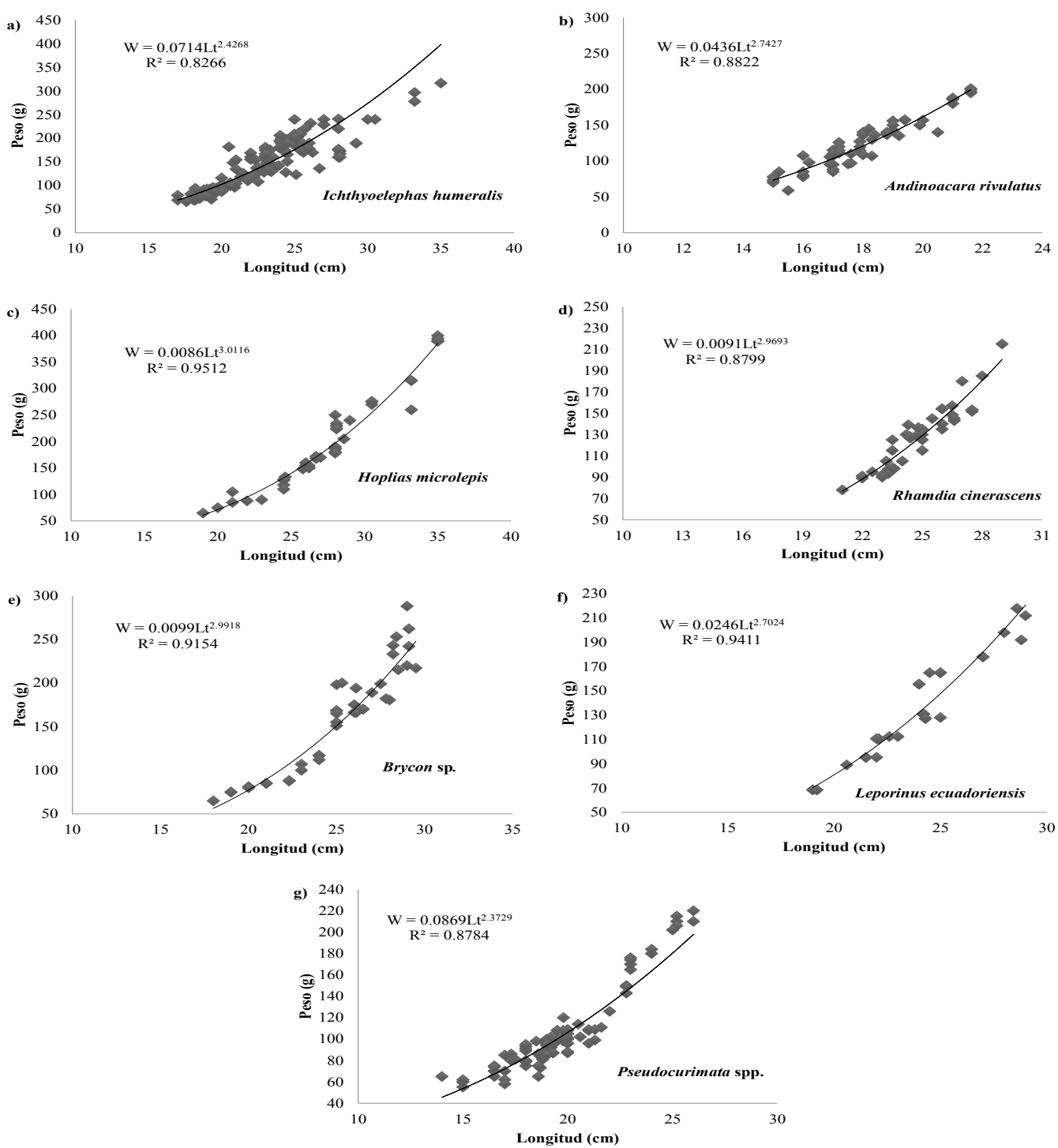

Figura 3. Relación longitud-peso de las principales especies de peces nativos capturados en el humedal Abras de Mantequilla, Ecuador, durante el 2015; a) Ichthyolephas humeralis, b) Andinoacara rivulatus, c) Hoplias microlepis, d) Rhamdia cinerascens, e) Brycon sp., f) Leporinus ecuadoriensis, g) Pseudocurimata spp. 
En el estudio existió una relación entre el tipo de crecimiento y la abundancia ya que especies que presentaron crecimiento alométrico negativo fueron las de mayor captura, mientras que las que presentaron crecimiento isométrico fueron menos abundantes (Figura 1; Cuadro 3). Esta relación se debe a la forma del cuerpo de la especie, ya que las especies que presentaron crecimiento isométrico (Hoplias microlepis, Rhamdia cinerascens, Brycon sp. Leporinus ecuadoriensis) tienen un cuerpo fusiforme y que a su vez podría facilitar el escape de las redes (Froese, 2006).

Vélez (1993) manifiesta que existen cambios gonadales mes a mes, los cuales están vinculados a las estaciones bien marcadas (lluviosa, transición y seca). Estos cambios concuerdan con lo registrado por Revelo (2010) en el sistema hídrico de la provincia Los Ríos. Conviene tener una relación estacional de la longitudpeso del pescado entero y eviscerado; esto puede ser de especial importancia en el caso del pescado entero, cuyo peso puede ser mucho mayor durante la época del desove que antes o después de la misma (FAO, 1974). Es necesario validar los resultados con datos morfométricos de peso eviscerado y longitud estándar para un mejor tratamiento estadístico.

\section{Conclusiones}

L a composición de las capturas estuvo mayormente representada por Ichthyolephas humeralis (Bocachico), especie autóctona y representativa de la provincia Los Ríos, seguida por Pseudocurimata spp. (Dica), mientras que Leporinus ecuadoriensis (Ratón) fue la especie menos capturada en el humedal Abras de Mantequilla. De las especies capturadas, solo Rhamdia cinerascens (Barbudo) presentó una talla media de captura de $24.81 \mathrm{~cm}$ de longitud total, inferior a la talla media de madurez $(25 \mathrm{~cm}$ Longitud total). En la mayoría de las especies de peces el reclutamiento ocurrió a un tamaño óptimo, pués, los peces aportaron con nuevos individuos a la población. La relación longitud-peso demostró que Ichthyolephas humeralis, Andinoacara rivulatus y Pseudocurimata spp., presentaron crecimiento alométrico negativo lo cual indica que las especies incrementan su longitud relativa más que su peso, mientras el resto de las especies tienen crecimiento isométrico que demuestra una ganancia o crecimiento igual en talla que en peso. Se evidenció que existe una relación entre las especies con crecimiento alométrico negativo y su abundancia en las capturas.

\section{Bibliografía}

Alvarez-Mieles, G., Irvine, K., Griensven, A. V., AriasHidalgo, M., Torres, A., \& Mynett, A. E. (2013). Relationships between aquatic biotic communities and water quality in a tropical river-wetland system (Ecuador). Environmental Science and Policy, 34 (december): 115-127.

Balboni, L., Colautti, D. C., \& Baigún, CRM. (2011). Biology of growth of Hoplias aff. malabaricus (Bloch, 1794) in a shallow pampean lake Argentina. Neotropical Ichthyology, 9(2): 437-444.

Barhill, B., \& López-León, E. (1973). Estudio sobre la biologia de peces del río Vínces. Guayaquil, Ecuador. Retrieved from http://www.institutopesca. gob.ec/programas-y-servicios/agua-dulce/

BirdLife International. (2016). Fichas de especies para migratorias neotropicales en las IBAs: Abras de Mantequilla (in Spanish). From http://www. birdlife.org

Boavida, M. J. (1999). Wetlands: Most relevant structural and functional aspects. Limnetica, 17, $57-63$.

Bru, S., Segura, F., \& Olaya, C. (2009). Crecimiento y mortalidad de la Liseta (Leporinus muyscorum, Steindachner, 1902) en el río Sinú, Colombia. MVZ Córdoba, 8(2): 340.

Cadena, M. (1981). Estudio preliminar de la relación longitud peso y etapas de madurez gonadal de Bocachico Ichthyoelephas humeralis. Guayaquil, Ecuador. Retrieved from http://www.institutopesca. gob.ec/programas-y-servicios/agua-dulce/

Chicaiza, D., \& Flores, H. (2016). Parámetros biológicos de Pseudocurimata boulengeri (Characiformes: Curimatidae) en el embalse Chongón, Ecuador. Revista de Biología Tropical, 64(March), 131-146

Cifuentes, R., González, J., Montoya, G., Jara, A., Ortíz, N., Piedra, P., \& Habit, E. (2012). Relación longitud-peso y factor de condición de los peces nativos del río San Pedro (cuenca del río Valdivia, Chile) . Gayana (Concepción) . JOUR, scielocl.

Corrêa, F., Huckembeck, S., \& Canziani, G. V. (2016). Length-weight relationship of Hoplias aff. malabaricus (Bloch, 1794) in a subtropical wetland . Brazilian Journal of Biology . JOUR, scielo .

Eigenmann, CH., \& Allen, WR. (1942). Fishes of western South America. I. The intercordilleran and Amazonian lowlands of Peru. II. The high pampas of Peru, Bolivia, and northern Chile. (University of Kentucky, Ed.). Lexington, Kentucky: Lexington, Ky., The University of Kentucky. Retrieved from https://archive.org/details/fishesofwesterns00eige 
FAO. (1974). Manual de Ciencia Pesquera Parte 2 - Métodos para Investigar los Recursos y su Aplicación. (M. Holden \& D. Raitt, Eds.). Roma, Italia: Organización de las naciones unidas para la agricultura y la alimentación (FAO). Retrieved from http://www.fao.org

FAO. (2004). Ordenación pesquera responsable en grandes ríos y embalses de América Latina: Informe del seminario Organización de las Naciones Unidas para la Agricultura y la Alimentación. FAO/ FishCode, 5(5): 78. Retrieved from http://www. fao.org

Fischer, W., Krupp, F., Schneider, W., Sommer, C., Carpenter, KE., \& Niem, VH. (1995). Guía FAO para La identificación de especies para los fines de La pesca Pacífico Centro Oriental (pp. 6471200). Roma, Italia: Organización de las naciones unidas para la agricultura y la alimentación (FAO). Retrieved from http://www.fao.org/docrep/010/ v6250s/v6250s00.htm

Florencio, A., \& Álvarez, G. (1983). Aspectos bioecológicos de los ríos Yaguachi y Milagro. Revita de Ciencias del Mar y Limnología, 2. Retrieved from http://www.institutopesca.gob.ec/ programas-y-servicios/agua-dulce/

Freitas, CE. de C., \& Rivas, AAF. (2006). A pesca e os recursos pesqueiros na amazônia ocidental. Ciência E Cultura, 58(3): 30-32.

Froese, R. (2006). Cube law, condition factor and weight-length relationships: History, metaanalysis and recommendations. Journal of Applied Ichthyology, 22(4): 241-253.

Gámez, D., Morón, E., \& Fuentes, J. (2014). Descripción del hábito alimentario de doce especies de peces asociados a la Ciénega Grande de Santa Marta, Colombia. Boletín de Investigaciones Marinas y Costeras - INVEMAR. JOUR. 43(1).

Granda, J., \& Montero, C. (2015). Aplicación de morfometría geométrica para la comparación de distintas poblaciones de Guanchiche (Hoplias spp.) en ecosistemas lénticos y lóticos del Ecuador. Escuela Superior Politécnica del Litoral. Retrieved from http://www.dspace.espol.edu.ec/

Hauser, M., \& Benedito, E. (2012). Species of the Hoplias aff malabaricus complex (Characiformes: Erythrinidae): An investigation of coexistence in a Neotropical floodplain. Zoologia (Curitiba), 29(1): 59-69.

Hilderbrand, R., \& Kershner, J. (2004). Influence of habitat type on food supply, selectivity, and diet overlap of Bonneville cutthroat trout and nonnative brook trout in Beaver Creek, Idaho. North American Journal of Fisheries Management, 24(1): 33-40.
Nava-Flores, A., \& Ancona, P. (2010). Peces nativos de agua dulce de América del Sur de interés para la acuicultura: Una síntesis del estado de desarrollo tecnológico de su cultivo. (A. FloresNava \& A. Brown, Eds.), FAO. Serie Acuicultura en Latinoamérica (1st ed., Vol. 1). Roma, Italia: Organización de las naciones unidas para la agricultura y la alimentación (FAO). Retrieved from http://www.fao.org/docrep/014/i1773s/i1773s.pdf

Pacheco, J. (2013). Aspectos pesqueros de las principales especies capturadas en el embalse parque lago chongón, durante 2013. Guayaquil, Ecuador. Retrieved from www.institutopesca.gob. ec

Pacheco, J. (2014). Aspectos Pesqueros de las Principales Especies Capturadas en el Embalse Parque Lago Chongón. Guayaquil, Ecuador. Retrieved from www.institutopesca.gob.ec

Pacheco, J. (2015). Aspectos Pesqueros de las Principales Especies Capturadas en el Embalse Parque Lago Chongón. Guayaquil, Ecuador. Retrieved from www.institutopesca.gob.ec

Pacheco, J., \& Cabanilla, C. (2013). Aspectos Biológicos $\mathrm{y}$ pesqueros de las principales especies capturadas en el embalse Chongón, durante 2012. Boletín Científico Técnico. Guayaquil, Ecuador. Retrieved from http://www.institutopesca.gob.ec

Prado, M., Revelo, W., Castro, R., Bucheli, R., Calderón, G., \& Macías, P. (2010). Caracterización química y biológica de sistemas hídricos en la provincia de los ríos-Ecuador. Guayaquil, Ecuador. Retrieved from http://www.institutopesca.gob.ec/programasy-servicios/agua-dulce/

Quevedo, O. (2008). Ficha Ramsar del Humedal Abras de Mantequilla - Ecuador 2008. Guayaquil, Ecuador. Retrieved from http://suia.ambiente.gob. ec

Revelo, W. (2010). Aspectos biológicos y Pesqueros de los principales peces del sistema hídrico de la provincia de Los Ríos, durante 2009 (Vol. 21). Guayaquil, Ecuador. Retrieved from http://www. institutopesca.gob.ec/programas-y-servicios/aguadulce/

Revelo, W., \& Castro, R. (2010). Aspectos biológicos $\mathrm{y}$ pesqueros de las principales especies de peces en el sistema hídrico de la provincia de Los Ríos, durante 2010. Guayaquil, Ecuador. Retrieved from www.institutopesca.gob.ec

Revelo, W., \& Elías, E. (2004). Aspectos biológicos de los principales recursos de aguas continentales, durante febrero y marzo del 2004 en la provincia de Los Ríos. Ciencias Marinas, 24-29. Retrieved from http://www.institutopesca.gob.ec/programasy-servicios/agua-dulce/ 
Revelo, W., \& Laaz, E. (2012). Catálogo de peces de aguas continentales de la provincia de Los RíosEcuador. Guayaquil, Ecuador. Retrieved from http://www.institutopesca.gob.ec

Ricker, WEE. (1975). Computation and interpretation of biological statistics of fish populations. Nature, (191), 401. http://doi.org/10.1038/108070b0

Rivera, JV. (2014). Ampliación de la distribución de Brycon dentex (Characiformes: Characidae) y Hamuelopsis elongatus (Perciformes: Heamulidae) en Ecuador. Avances En Ciencias e Ingenierías, 6(2): 17-18. Retrieved from http://www.usfq.edu. ec/

dos Santos, ALB., Pessanha, ALM., da Costa, MR. \& Araújo, FG. (2004). Relação peso-comprimento de Orthopristis ruber (Cuvier) (Teleostei, Haemulidae) na Baia de Sepetiba, Rio de Janeiro, Brasil . Revista Brasileira de Zoologia . JOUR, scielo .
Vélez, C. (1993). Ciclo reproductivo y talla de la primera madurez sexual en hembras de guanchiche, hoplias microlepis (gunther). Rio Vinces - Ecuador. Revista de Ciencias Del Mar, 3(1), 227. Retrieved from http://www.institutopesca.gob.ec/

Villón, C., Gaibor, N., Bohorquez, H., \& Santamaría, J. (1999). Problemática de la pesca artesanal en el río Babahoyo, terrenos inundados adyacentes y afluentes: estrategias alternativas de manejo. Guayaquil, Ecuador. Retrieved from http://www. institutopesca.gob.ec/programas-y-servicios/aguadulce/

Zar, JH. (2010). Biostatistical Analysis. (P. Hall, Ed.) (5th ed.). Book, Pearson. 